T. Hiramatsu

Nagoya Math. J.

Vol. 105 (1987), 169-186

\title{
ON SOME DIMENSION FORMULA FOR AUTOMORPHIC FORMS OF WEIGHT ONE, II
}

\author{
TOYOKAZU HIRAMATSU
}

\section{§. Introduction}

Let $\Gamma$ be a fuchsian group of the first kind and assume that $\Gamma$ contains the element $\left(\begin{array}{rr}-1 & 0 \\ 0 & -1\end{array}\right)(=-I)$, and let $\chi$ be a unitary representation of $\Gamma$ of degree 1 such that $\chi(-I)=-1$. Let $S_{1}(\Gamma, \chi)$ be the linear space of cusp forms of weight one on the group $\Gamma$ with character $\chi$. We shall denote by $d_{1}$ the dimension of the linear space $S_{1}(\Gamma, \chi)$. It is not effective to compute the number $d_{1}$ by means of the Riemann-Roch theorem. Because of this reason, it is an interesting problem in its own right to determine the number $d_{1}$ by some other method (for example, [5]).

When the group $\Gamma$ has a compact fundamental domain in the upper half plane $S^{1)}$, we have obtained the following dimension formula which is a slightly modified form of the previous result ([1]):

$$
d_{1}=\frac{1}{2} \sum_{\{M\}} \frac{\chi(M)}{[\Gamma(M): \pm I]} \frac{\bar{\zeta}}{1-\bar{\zeta}^{2}}+\frac{1}{2} \operatorname{Res}_{s=0} \zeta^{*}(s),,^{2)}
$$

where the sum over $\{M\}$ is taken over the distinct elliptic conjugacy classes of $\Gamma /\{ \pm I\}, \Gamma(M)$ denotes the centralizer of $M$ in $\Gamma, \bar{\zeta}$ is one of the eigenvalues of $M$, and $\zeta^{*}(s)$ denotes the Selberg type zeta-function defined by

$$
\zeta^{*}(s)=\sum_{\alpha=1}^{\infty} \sum_{k=1}^{\infty} \frac{\chi\left(P_{\alpha}\right)^{k} \log \lambda_{0, \alpha}}{\lambda_{0, \alpha}^{k}-\lambda_{0, \alpha}^{-k}}\left|\lambda_{0, \alpha}^{k}+\lambda_{0, \alpha}^{-k}\right|^{-s} .
$$

Here, $\lambda_{0, \alpha}$ denotes the eigenvalue $\left(\lambda_{0, \alpha}>1\right)$ of a representative $P_{\alpha}$ of the primitive hyperbolic conjugacy classes $\left\{P_{\alpha}\right\}$ in $\Gamma /\{ \pm I\}$.

Received November 9, 1985.

Revised September 12, 1986.

1) In this case, $S_{1}(\Gamma, \chi)$ denotes simply the space of all holomorphic automorphic forms of weight one with $\chi$.

2) For this modified formula of $d_{1}$, refer to Hiramatsu ([6], Remark 1 in $\S 2$ ). 
The purpose of this paper is to give a similar formula of the number $d_{1}$ when the group $\Gamma$ is of finite type reduced at infinity and $\chi^{2} \neq 1$, by using the method of Selberg ([3], [4]). In this case, the operator $\omega_{\delta}$ in [1] is not generally completely continuous on the space $L^{2}(\Gamma \backslash \tilde{S}, \chi)$ and a new term from cusp ought to have added to the formula (1). The notation used here will generally be those of [1].

It is a pleasure to record my thanks to Professor H. Shimizu for his advice and encouragement during the writing up.

\section{$\S 1$. The Selberg eigenspace $\mathfrak{M}(1,-3 / 2, \chi)$ for a group $\Gamma$ of finite type}

Let $\Gamma$ be a fuchsian group of the first kind containing the element $-I$, and suppose that $\Gamma$ has a non-compact fundamental domain in $S$. Let $T$ be the real torus and put $\tilde{S}=S \times T$. Denote by $L^{2}(\Gamma \backslash \tilde{S}, \chi)$ the following set

$$
\left\{f \in L^{2}(\Gamma \mid \tilde{S}): f(g(z, \phi))=\chi(g) f(z, \phi) \text { for all } g=\left(\begin{array}{ll}
a & b \\
c & d
\end{array}\right) \in \Gamma\right\},
$$

where

$$
f(g(z, \phi))=f\left(\frac{a z+b}{c z+d}, \phi+\arg (c z+d)\right) .
$$

Moreover we denote by $\mathfrak{M}_{\Gamma}(k, \lambda, \chi)=\mathfrak{M}(k, \lambda, \chi)$ the set of all functions $f(z, \phi)$ satisfying the following conditions:

(i) $f(z, \phi) \in L^{2}(\Gamma \backslash \tilde{S}, \chi)$,

(ii) $\widetilde{\Delta} f(z, \phi)=\lambda f(z, \phi), \frac{\partial}{\partial \phi} f(z, \phi)=-i k f(z, \phi)$.

Then we have the following

Lemma. To each function $f(z, \phi) \in \mathfrak{M}(1, \lambda, \chi)$, we associate a function on S by letting

$$
F(z)=e^{i \phi} y^{-1 / 2} f(z, \phi) .
$$

Then the function $F(z)$ belongs to $S_{1}(\Gamma, \chi)$ if and only if

$$
f(z, \phi) \in \mathfrak{M}(1,-3 / 2, \chi) \text {. }
$$

Proof. For each $F(z) \in S_{1}(\Gamma, \chi)$ we define $f(z, \phi)$ on $\tilde{S}$ by

$$
f(z, \phi)=e^{-i \phi} y^{1 / 2} F(z) .
$$

Then the function $f(z, \phi)$ satisfies the conditions: 
(1.2) $f(g(z, \phi))=\chi(g) f(z, \phi)$ for all $g \in \Gamma$;

(1.3) $(\partial / \partial \phi) f(z, \phi)=-i f(z, \phi)$;

(1.4) $\widetilde{\Delta} f(z, \phi)=-(3 / 2) f(z, \phi)$ by regularity of $F(z)$ on $S$;

(1.5) Since $y^{1 / 2}|F(z)|$ is bounded on $S$,

$$
\begin{aligned}
\|f\| & =\frac{1}{\pi} \int_{\Gamma \backslash \bar{s}}\left|e^{-i \phi} y^{1 / 2} F(z)\right|^{2} \frac{d x d y d \phi}{y^{2}} \\
& =\int_{\Gamma \backslash S}\left|y^{1 / 2} F(z)\right|^{2} \frac{d x d y}{y^{2}}<\infty .
\end{aligned}
$$

Therefore, by $(1.2) \sim(1.5)$, the function $f(z, \phi)$ belongs to $\mathfrak{M}(1,-3 / 2, \chi)$. We now prove conversely that any function in $\mathfrak{M}(1,-3 / 2, \chi)$ must be of the form (1.1) with $F(z) \in S_{1}(\Gamma, \chi)$. Let $f(z, \phi)$ be a function in $\mathfrak{M}(1,-3 / 2, \chi)$. Put

$$
F(z)=e^{i \phi} y^{-1 / 2} f(z, \phi) .
$$

Then the $\Gamma$-invariance of $f(z, \phi)$ is equivalent to a transformation law for $F(z)$ :

$$
F(g(z))=\chi(g)(c z+d) F(z)
$$

for all $g=\left(\begin{array}{ll}a & b \\ c & d\end{array}\right) \in \Gamma$. Therefore, it is sufficient for the proof of the latter half of our lemma to show that $F(z)$ is holomorphic with respect to the complex variable $z$ on $S$, and vanishes at every cusp of $\Gamma$.

Let $g$ be the Lie algebra of $S L_{2}(R)(=G)$. Then we can take the basis $\mathfrak{a}$ of $\mathfrak{g}$ such that the Lie derivatives associated with the elements of $a$ are given by the following invariant differential operators:

$$
\begin{aligned}
& X=y \cos 2 \phi \frac{\partial}{\partial x}-y \sin 2 \phi \frac{\partial}{\partial y}+\frac{1}{2}(\cos 2 \phi-1) \frac{\partial}{\partial \phi}, \\
& Y=y \sin 2 \phi \frac{\partial}{\partial x}+y \cos 2 \phi \frac{\partial}{\partial y}+\frac{1}{2} \sin 2 \phi \frac{\partial}{\partial \phi}, \\
& \Phi=\frac{\partial}{\partial \phi} .
\end{aligned}
$$

It is easy to see that

$$
\widetilde{\Delta}=\left(X+\frac{1}{2} \Phi\right)^{2}+Y^{2}+\Phi^{2}
$$

Now we put 


$$
A^{-}=2\left(X+\frac{1}{2} \Phi\right)+2 i Y
$$

Then, the function $F(z)$ is holomorphic on $S$ if and only if

$$
A^{-} f(z, \phi)=0 \text {. }
$$

To prove (1.6), first note that the operation of $A^{-}$depends only on the representations of the Lie algebra g. Let $L_{d}^{2}(\Gamma \backslash G, \chi)$ be the discrete part of the space $L^{2}(\Gamma \backslash G, \chi)$. Then $f \in L_{d}^{2}(\Gamma \backslash G, \chi)$. Let

$$
L_{d}^{2}(\Gamma \backslash G, \chi)=\sum_{i} V_{i}
$$

be the irreducible splitting of the space $L_{d}^{2}(\Gamma \backslash G, \chi)$ and put

$$
f=\sum_{i} f_{i} \quad\left(f_{i} \in V_{i}\right) .
$$

Then, if $f_{i} \neq 0$, we have

$$
\widetilde{\Delta} f_{i}=-\frac{3}{2} f_{i}, \frac{\partial}{\partial \phi} f_{i}=-\sqrt{-1} f_{i}
$$

Therefore, each subspace $V_{i}$ such that $f_{i} \neq 0$ is isomorphic to the space $H_{1}$ of the irreducible representation of the limit of discrete series. Hence it is sufficient for the proof of (1.6), to show that for any highest weight vector $\varphi$ in $H_{1}$,

$$
A^{-} \varphi=0 \text {. }
$$

For example, by Lemma 5.6 in [2], the formula (1.7) is well known.

Next we shall see the condition for $F(z)$ at every cusp of $\Gamma$. Let $s$ be a cusp of $\Gamma \cap \operatorname{Ker} \chi$. We may assume that $s=\infty$ and the intersection of a fundamental domain for $\Gamma$ and a neighborhood of $\infty$ is the following type

$$
\{z=x+i y: 0 \leqq x \leqq 1, y \geqq M\},
$$

where $M$ denotes a positive constant. Then, by the given condition $f(z, \phi) \in L^{2}(\Gamma \backslash \tilde{S}, \chi)$

$$
\int_{M}^{\infty}\left\{\int_{0}^{1} y|F(z)|^{2} d x\right\} \frac{d y}{y^{2}}<\infty
$$

Let

$$
F(z)=\sum_{n=-\infty}^{\infty} a_{n} e^{2 \pi i n z}
$$


be the Fourier expansion of $F$ at $\infty$. Then, we have

$$
\begin{aligned}
\int_{0}^{1}|F(z)|^{2} d x & =\int_{0}^{1}\left(\sum_{n} a_{n} e^{2 \pi i n z}\right)\left(\sum_{m} \bar{a}_{m} e^{-2 \pi i m \bar{z}}\right) d x \\
& =\sum_{n, m} a_{n} \bar{a}_{m} \int_{0}^{1} e^{2 \pi i(n-m) x-2 \pi(n+m) y} d x \\
& =\sum_{n}\left|a_{n}\right|^{2} e^{-4 \pi n y} .
\end{aligned}
$$

Therefore,

$$
\int_{M}^{\infty} y\left(\sum_{n}\left|a_{n}\right|^{2} e^{-4 \pi n y}\right) \frac{d y}{y^{2}}=\sum_{n}\left|a_{n}\right|^{2} \int_{M}^{\infty} y^{-1} e^{-4 \pi n y} d y
$$

If $n \leqq 0$, then

$$
\int_{M}^{\infty} y^{-1} e^{-4 \pi n y} d y=\infty,
$$

so that $a_{n}=0$ for all $n \leqq 0$.

Q.E.D.

\section{§2. Eisenstein series and continuous spectrum}

2.1. This section is essentially based upon the work of Selberg ([3], [4]). We shall review the definition and elementary properties of Eisenstein series for the cusp $\infty$, and the spectral decomposition of $L^{2}(\Gamma \backslash \tilde{S}, \chi)$ (abbreviated hereafter as $\left.L^{2}(\Gamma \backslash \tilde{S})\right)$. Let $\Gamma$ be of finite type reduced at $\infty$, namely, $\infty$ is a cusp of $\Gamma$ and the stabilizer $\Gamma_{\infty}$ of $\infty$ in $\Gamma$ is equal to $\pm \Gamma_{0}$ with $\Gamma_{0}=\left\{\left(\begin{array}{rr}1 & m \\ 0 & 1\end{array}\right): m \in Z\right\}$. The Eisenstein series $E_{x}(z, \phi ; s)$ attached to the cusp $\infty$ and $\chi$ is then defined by

$$
E_{\chi}(z, \phi ; s)=\sum_{\substack{M \in \Gamma_{\infty} \infty \Gamma \\
M=\left(\begin{array}{c}
* * \\
c \neq d
\end{array}\right)}} \frac{\bar{\chi}(M) y^{s}}{|c z+d|^{2 s}} e^{-i(\phi+\arg (c z+d))},
$$

where $s=\sigma+i r$ with $\sigma>1$. It is easy to check that

(i ) $E_{\mathrm{x}}(M(z, \phi) ; s)=\chi(M) E_{\mathrm{x}}(z, \phi ; s)$ for $M \in \Gamma$;

(ii) $\bar{\Delta} E_{x}(z, \phi ; s)=\left\{s(s-1)-\frac{5}{4}\right\} E_{x}(z, \phi ; s)$;

(iii) $\frac{\partial}{\partial \phi} E_{\chi}(z, \phi ; s)=-i E_{x}(z, \phi ; s)$.

Since $E_{\mathrm{x}}(z+1, \phi ; s)=E_{\chi}(z, \phi ; s)$, we have a Fourier-Bessel expansion of the form

$$
E_{\chi}(z, \phi ; s)=\sum_{m=-\infty}^{\infty} a_{m, x}(y, \phi ; s) e^{2 \pi i m x}
$$


where

$$
a_{m, \chi}(y, \phi ; s)=\int_{0}^{1} E_{\chi}(z, \phi ; s) e^{-2 \pi i m x} d x .
$$

Let us now try to find the constant term $a_{0, x}(y, \phi ; s)$ explicitly. Put

$$
a_{0, x}(y, \phi ; s)=e^{-i \phi} a_{0, x}(y ; s),
$$

and

$$
E_{\chi}(z ; s)=e^{i \phi} E_{\chi}(z, \phi ; s) \text {; }
$$

then

$$
\begin{aligned}
& a_{0, \chi}(y ; s)=\int_{0}^{1} E_{\chi}(z ; s) d x \\
& =\int_{\substack{0 \\
0 \\
M \in \Gamma_{\infty} \backslash \Gamma \\
M=(* *) \\
c>0}} \frac{\bar{\chi}(M) y^{s}}{|c z+d|^{2 s}} \lambda(c z+d)^{-1} d x \quad\left(\lambda(z)=\frac{z}{|z|}, z \neq 0\right) \\
& =y^{s}+\int_{\substack{0 \\
M \in \Gamma_{\infty}^{\infty} \backslash \Gamma \\
M=d \\
c>d \\
c>0}} \frac{\bar{\chi}(M) y^{s}}{|c z+d|^{2 s}} \lambda(c z+d)^{-1} d x
\end{aligned}
$$

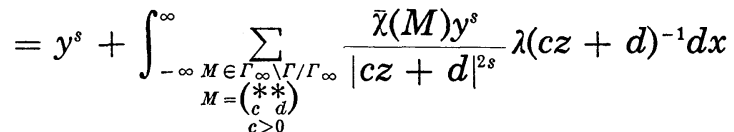

$$
\begin{aligned}
& =y^{s}+\sum_{\substack{c>0 \\
d \text { mod } \\
\left(\begin{array}{c}
* * \\
c
\end{array}\right.}} \frac{\bar{\chi}(c, d)}{|c|^{2 s}} \int_{-\infty}^{\infty} \frac{y^{s}}{\left|z+\frac{d}{c}\right|^{2 s}} \lambda\left(z+\frac{d}{c}\right)^{-1} d x \\
& =y^{s}+y^{s} \varphi_{0, x}(s) \int_{-\infty}^{\infty} \frac{\lambda(z)^{-1}}{|z|^{2 s}} d x \\
& =y^{s}+y^{1-s} \varphi_{0, x}(s) \int_{-\infty}^{\infty} \frac{\lambda(i+t)^{-1}}{\left(1+t^{2}\right)^{s}} d t,
\end{aligned}
$$

where

$$
\varphi_{0, x}(s)=\sum_{\substack{c>0 \\
d \bmod c \\
\left(\begin{array}{l}
* * * \\
c
\end{array}\right) \in \Gamma}} \frac{\bar{\chi}(c, d)}{|c|^{2 s}}
$$

Furthermore,

$$
\begin{aligned}
\int_{-\infty}^{\infty} \frac{\lambda(i+t)^{-1}}{\left(1+t^{2}\right)^{s}} d t & =\int_{0}^{\pi} \frac{(i+\cot \theta)^{-1}}{|\operatorname{cosec} \theta|^{2 s-1}} \operatorname{cosec}^{2} \theta d \theta \\
& =\int_{0}^{\pi} \sin ^{2 s-2} \theta(\cos \theta-i \sin \theta) d \theta
\end{aligned}
$$




$$
\begin{aligned}
& =-i \int_{0}^{\pi} \sin ^{2 s-1} \theta d \theta \\
& =-i \sqrt{\pi} \frac{\Gamma(s)}{\Gamma\left(s+\frac{1}{2}\right)} .
\end{aligned}
$$

Therefore we have

$$
a_{0, \mathrm{x}}(y ; s)=y^{s}-i \sqrt{\pi} \frac{\Gamma(s)}{\Gamma\left(s+\frac{1}{2}\right)} \varphi_{0, x}(s) y^{1-s} .
$$

2.2. Since the group $\Gamma$ is reduced at $\infty$, the integral operator $\omega_{\delta}$ in [1] is not generally completely continuous on $L^{2}(\Gamma \backslash \tilde{S})$ and beside the discrete spectrum in $L^{2}(\Gamma \backslash \tilde{S})$, the operator $\omega_{\delta}$ has one or more continuous spectra in $L^{2}(\Gamma \backslash \tilde{S})$. The space $L^{2}(\Gamma \backslash \tilde{S})$ has the following spectral decomposition

$$
L^{2}(\Gamma \backslash \tilde{S})=L_{0}^{2}(\Gamma \backslash \tilde{S}) \oplus L_{s p}^{2}(\Gamma \backslash \tilde{S}) \oplus L_{c o n t}^{2}(I \backslash \tilde{S}),
$$

where $L_{0}^{2}$ is the space of cusp forms and is discrete, $L_{s p}^{2}$ is the discrete part of the orthogonal complement of $L_{0}^{2}$, and $L_{\text {cont }}^{2}$ is continuous part of the spectrum, In the following we shall only consider the case

$$
\chi\left(\left(\begin{array}{ll}
1 & 1 \\
0 & 1
\end{array}\right)\right)=1
$$

By using the analytic continuation of the Eisenstein series (2.1) as a function of $s$ for $s=1 / 2+i r$, we put

$$
\tilde{H}_{\delta}\left(z, \phi ; z^{\prime}, \phi^{\prime}\right)=\frac{1}{4 \pi^{2}} \int_{-\infty}^{\infty} h(r) E_{\chi}\left(z, \phi ; \frac{1}{2}+i r\right) \overline{E_{\chi}\left(z^{\prime}, \phi^{\prime} ; \frac{1}{2}+i r\right)} d r
$$

where $h(r)$ denotes the eigenvalue of $\omega_{\delta}$ in $\mathfrak{M}(1, \lambda, \chi)([1], \mathrm{p} .217)$ :

$$
h(r)=2^{2+\delta} \pi \frac{\Gamma\left(\frac{1}{2}\right) \Gamma\left(\frac{1+\delta}{2}\right)}{\Gamma(\delta) \Gamma\left(1+\frac{\delta}{2}\right)} \Gamma\left(\frac{\delta}{2}+i r\right) \Gamma\left(\frac{\delta}{2}-i r\right)
$$

with $\lambda=s(s-1)-5 / 4$ and $s=1 / 2+i r$. Then the integral operator $\tilde{K}_{\delta}=$ $K_{\delta}-\tilde{H}_{\delta}$ is now completely continuous on $L^{2}(\Gamma \backslash \tilde{S})$ and has all discrete spectra of $K_{\delta}$, where

$$
K_{\delta}\left(z, \phi ; z^{\prime}, \phi^{\prime}\right)=\underset{M \in \Gamma /\{ \pm I\}}{2 \sum_{1}} \chi(M) \omega_{\delta}\left(z, \phi ; M\left(z^{\prime}, \phi^{\prime}\right)\right) .
$$


Furthermore, an eigenvalue of $f(z, \phi)$ in $L_{0}^{2}(\Gamma \backslash \tilde{S}) \oplus L_{\mathrm{sp}}^{2}(\Gamma \backslash \tilde{S})$ for $\tilde{K}_{\delta}$ is equal to that for $K_{\delta}$ and the image of $\tilde{K}_{\delta}$ on it is contained in $L_{0}^{2}(\Gamma \backslash \tilde{S})$. Considering the trace of $\tilde{K}_{\delta}$ on $L_{0}^{2}(\Gamma \backslash \tilde{S})$, we now obtain the following modified trace formula

$$
\begin{aligned}
\sum_{n=1}^{\infty} h\left(\lambda^{(n)}\right) & =\int_{\tilde{D}} \tilde{K}_{\delta}(z, \phi ; z, \phi) d(z, \phi) \\
& =\int_{\tilde{D}}\left\{\underset{M \in \Gamma /\{ \pm I\}}{2 \sum_{i}} \chi(M) \omega_{\delta}(z, \phi ; M(z, \phi))-\tilde{H}_{\delta}(z, \phi ; z, \phi)\right\} d(z, \phi),
\end{aligned}
$$

where $\tilde{D}$ denotes a fundamental domain of $\Gamma$ in $\tilde{S}$, and each of $\lambda^{(n)}$ denotes an eigenvalue corresponding to an orthogonal basis $\left\{f^{(n)}\right\}$ for $L_{0}^{2}(\Gamma \backslash \tilde{S})$.

\section{§3. A formula for the dimension $d_{1}$}

The purpose of this section is to obtain an explicit formula for the dimension $d_{1}$ by calculating the integrals in (2.3). We put

$$
\begin{gathered}
\int_{\tilde{D}}\left\{\underset{M \in \Gamma /\{ \pm I\}}{2 \sum} \chi(M) \omega_{\delta}(z, \phi ; M(z, \phi))-\tilde{H}_{\delta}(z, \phi ; z, \phi)\right\} d(z, \phi) \\
=J(I)+J(P)+J(R)+J(\infty),
\end{gathered}
$$

where $J(I), J(P), J(R)$, and $J(\infty)$ denote respectively the identity component, the hyperbolic component, the elliptic component, and the parabolic component of the traces. Then the components $J(I), J(P)$, and $J(R)$ were obtained already in [1] and [6]. So in the following we shall calculate the component $J(\infty)$. Since $\Gamma$ is reduced at $\infty$, the set $\Gamma /\{ \pm I\}-\{I\}$ gives a complete system of representatives of the parabolic conjugacy classes in $\Gamma /\{ \pm I\}$ and for each $\gamma \in \Gamma_{\infty}-\{ \pm I\}$, the stabilizer of $\gamma$ in $\Gamma$ is always equal to $\Gamma_{\infty}$. Put

$$
P_{\Gamma}=\left\{\gamma^{-1}\left(\begin{array}{rr}
1 & m \\
0 & 1
\end{array}\right) \gamma: m \in Z, m \neq 0, \gamma \in \Gamma_{\infty} \backslash \Gamma\right\} .
$$

Then $P_{\Gamma}$ is the set consisting of all parabolic elements in $\Gamma /\{ \pm I\}$. Hence

$$
\begin{aligned}
& \int_{\tilde{D}} \sum_{\substack{M \in \Gamma / \pm I\} \\
M: \text { parabolic }}} \chi(M) \omega_{\delta}(z, \phi ; M(z, \phi)) d(z, \phi)=\sum_{\substack{M \in \Gamma /\{+I\} \\
M: \text { parabolic }}} \int_{\tilde{D}} \chi(M) \omega_{\delta}(z, \phi ; M(z, \phi)) d(z, \phi) \\
& \quad=\sum_{\substack{M \in \Gamma_{0} \\
M \neq I}} \sum_{r \in \Gamma_{\infty} \backslash \Gamma} \int_{\tilde{D}} \omega_{\delta}(\gamma(z, \phi) ; M r(z, \phi)) d(z, \phi) \\
& \quad=\sum_{\substack{M \in \Gamma_{0} \\
M \neq I}} \int_{\tilde{D}_{\Gamma_{\infty}}} \omega_{\delta}(z, \phi ; M(z, \phi)) d(z, \phi)
\end{aligned}
$$


where $\tilde{D}_{\Gamma_{\infty}}$ denotes a fundamental domain of $\Gamma_{\infty}$ in $\tilde{S}$. Therefore we have

$$
\begin{aligned}
J(\infty) & =\lim _{Y \rightarrow \infty}\left\{\int_{0}^{Y} \int_{0}^{1} \int_{0}^{\pi} 2 \int_{0}^{\pi \in I_{0}} \omega_{\delta}(z, \phi ; M(z, \phi)) d(z, \phi)\right. \\
& \left.-\int_{\tilde{D}_{Y}} \tilde{H}_{\delta}(z, \phi ; \boldsymbol{z}, \phi) d(\boldsymbol{z}, \phi)\right\},
\end{aligned}
$$

where $\tilde{D}_{Y}=\tilde{D}-\tilde{D}_{Y}^{\prime}$ with the direct product $\tilde{D}_{Y}^{\prime}$ of the real torus $T$ and the subdomain of the strip determined by $\operatorname{Im} z>Y$ for a sufficiently large $Y>0$. Furthermore

(A)

$$
\begin{aligned}
& \int_{0}^{Y} \int_{0}^{1} \int_{0}^{\pi} 2 \sum_{\substack{M \in \Gamma_{0} \\
M \neq I}} \omega_{\delta}(z, \phi ; M(z, \phi)) d(z, \phi) \\
& \quad=2 \sum_{\substack{m \in \mathbb{Z} \\
m \neq 0}} \int_{0}^{Y} \int_{0}^{1} \int_{0}^{\pi} \omega_{\delta}\left(z, \phi ; M_{0}^{m}(z, \phi)\right) d(z, \phi) \quad\left(M_{0}=\left(\begin{array}{ll}
1 & 1 \\
0 & 1
\end{array}\right)\right) \\
& \quad=2 \pi \sum_{\substack{m \in Z \\
m \neq 0}} \int_{0}^{Y}\left|\frac{y}{\frac{2 y i-m}{2 i}}\right|^{\delta} \frac{y}{\frac{2 y i-m}{2 i}} \frac{d y}{y^{2}} \\
& \quad=2^{\delta+4} \pi \sum_{m=1}^{\infty} \int_{0}^{Y} \frac{1}{\left(4+(m / y)^{2}\right)^{\delta / 2+1}} \frac{d y}{y^{2}}
\end{aligned}
$$

If we put

$$
k(t)=\frac{1}{(4+t)^{\delta / 2+1}} \quad \text { and } f(x)=2 \int_{0}^{Y} k\left(\frac{x^{2}}{y^{2}}\right) \frac{d y}{y^{2}}
$$

then

$$
f(x)=\frac{1}{x} \int_{x^{2} / Y^{2}}^{\infty} \frac{k(t)}{\sqrt{t}} d t
$$

and hence

$$
\int_{0}^{Y} \int_{0}^{1} \int_{0}^{\pi} 2 \sum_{\substack{M \in \Gamma_{0} \\ M \neq I}} \omega_{\delta}(z, \phi ; M(z, \phi)) d(z, \phi)=2^{\delta+3} \pi \sum_{m=1}^{\infty} f(m) .
$$

Now we make use of a summation formula due to Euler-MacLaurin:

$$
\sum_{m=1}^{\infty} f(m)=\frac{1}{2} f(1)+\int_{1}^{\infty} f^{\prime}(x)\{x\} d x+\int_{1}^{\infty} f(x) d x
$$

where $[x]$ denotes the greatest integer in $x$ and $\{x\}=x-[x]-1 / 2$. Then we have 


$$
\frac{1}{2} f(1) \longrightarrow 2^{-(\delta+2)} \frac{\Gamma\left(\frac{1}{2}\right) \Gamma\left(\frac{\delta+1}{2}\right)}{\Gamma\left(1+\frac{\delta}{2}\right)},
$$

and

$$
\int_{1}^{\infty} f^{\prime}(x)\{x\} d x=2^{-(\delta+1)} \frac{\Gamma\left(\frac{1}{2}\right) \Gamma\left(\frac{\delta+1}{2}\right)}{\Gamma\left(1+\frac{\delta}{2}\right)}\left(C-\frac{1}{2}\right)+o(1),
$$

as $Y \rightarrow \infty$, where $C$ is Euler's constant. As for the third integral of (3.1), we have first the following by [3]:

$$
\begin{aligned}
& \int_{1}^{\infty} f(x) d x \\
& \quad=2^{-(\delta+1)} \frac{\Gamma\left(\frac{1}{2}\right) \Gamma\left(\frac{\delta+1}{2}\right)}{\Gamma\left(1+\frac{\delta}{2}\right)} \log Y+\frac{1}{2} \int_{0}^{\infty} \frac{\log t \cdot k(t)}{\sqrt{t}} d t+o(1),
\end{aligned}
$$

as $Y \rightarrow \infty$. Furthermore,

$$
\begin{aligned}
\frac{1}{2} \int_{0}^{\infty} \frac{\log t \cdot k(t)}{\sqrt{t}} d t=\frac{1}{2} \int_{0}^{\infty} \frac{\log t}{\sqrt{t}(4+t)^{\delta / 2+1}} d t \\
=2^{-(\delta+1)} \log 2 \int_{0}^{\infty} \frac{1}{\sqrt{t}(1+t)^{\delta / 2+1}} d t+2^{-(\delta+2)} \int_{0}^{\infty} \frac{\log t}{\sqrt{t}(1+t)^{\delta / 2+1}} d t \\
=2^{-(\delta+1)} \log 2 \frac{\Gamma\left(\frac{1}{2}\right) \Gamma\left(\frac{\delta+1}{2}\right)}{\Gamma\left(1+\frac{\delta}{2}\right)} \\
\quad+2^{-(\delta+2)}\left\{-\sum_{k=0}^{\infty}\left(\begin{array}{c}
-\delta / 2-1 \\
k
\end{array}\right) \frac{1}{\left(\frac{1}{2}+k\right)^{2}}+\sum_{k=0}^{\infty}\left(\begin{array}{c}
-\delta / 2-1 \\
k
\end{array}\right) \frac{1}{\left(\frac{\delta+9}{2}+k\right)^{2}}\right\},
\end{aligned}
$$

where $-2<\delta<4$. Summing up the above results, we obtain for the first half of $J(\infty)$,

$$
\int_{0}^{Y} \int_{0}^{1} \int_{0}^{\pi} 2 \sum_{\substack{M \in \Gamma_{0} \\ M \neq I}} \omega_{\delta}(z, \phi ; M(z, \phi)) d(z, \phi)
$$




$$
\begin{aligned}
& =2^{2} \pi \frac{\Gamma\left(\frac{1}{2}\right) \Gamma\left(\frac{\delta+1}{2}\right)}{\Gamma\left(1+\frac{\delta}{2}\right)}(\log Y+C+\log 2) \\
& +2 \pi\left\{-\sum_{k=0}^{\infty}\left(\begin{array}{c}
-\delta / 2-1 \\
k
\end{array}\right) \frac{1}{\left(\frac{1}{2}+k\right)^{2}}\right. \\
& \left.+\sum_{k=0}^{\infty}\left(\begin{array}{c}
-\delta / 2-1 \\
k
\end{array}\right) \frac{1}{\left(\frac{\delta+9}{2}+k\right)^{2}}\right\}+o(1),
\end{aligned}
$$

as $Y \rightarrow \infty$.

(B) We define the following the compact part of $E_{x}(z, \phi ; s)$ :

$$
\tilde{E}_{x}(z, \phi ; s)= \begin{cases}E_{\chi}(z, \phi ; s) & \text { for } y \leqq Y, \\ E_{\chi}(z, \phi ; s)-e^{-i \phi}\left(y^{s}+\varphi_{x}(s) y^{1-s}\right) & \text { for } y>Y,\end{cases}
$$

with $\varphi_{x}(s)=-i \sqrt{\pi} \frac{\Gamma(s)}{\Gamma\left(s+\frac{1}{2}\right)} \varphi_{0, x}(s)$. Then,

$$
\begin{aligned}
\int_{\tilde{D}_{Y}} \tilde{H}_{\delta}(z, \phi ; z, \phi) d(z, \phi) \\
\quad=\frac{1}{4 \pi^{2}} \int_{\tilde{D}_{Y}} \int_{-\infty}^{\infty} h(r) E_{\chi}\left(z, \phi ; \frac{1}{2}+i r\right) \overline{E_{\chi}\left(z, \phi ; \frac{1}{2}-i r\right)} d r d(z, \phi) \\
\quad=\frac{1}{4 \pi^{2}} \lim _{\sigma \rightarrow \frac{1}{2}} \int_{\tilde{D}} \int_{-\infty}^{\infty} h(r)|\tilde{E}(z, \phi ; \sigma+i r)|^{2} d r d(z, \phi)+o(1)
\end{aligned}
$$

as $Y \rightarrow \infty$. Moreover,

$$
\begin{aligned}
& \left.\int_{\tilde{D}} \int_{-\infty}^{\infty} h(r)\left|\tilde{E}_{x}(z, \phi ; s)\right|^{2} d r d(z, \phi)=\int_{\tilde{D}_{Y}} \int_{-\infty}^{\infty} h(r) E_{x}(z, \phi ; s) \overline{E_{\chi}(z, \phi ; s}\right) d r d(z, \phi) \\
& +\int_{\tilde{D}_{Y^{\prime}}} \int_{-\infty}^{\infty} h(r)\left\{E_{\chi}(z, \phi ; s)-e^{-i \phi}\left(y^{s}+\varphi_{\chi}(s) y^{1-s}\right)\right\} \\
& \times\left\{\overline{E_{\chi}(z, \phi ; s)}-e^{i \phi}\left(y^{\bar{s}}+\overline{\varphi_{\chi}(s)} y^{1-\bar{s}}\right)\right\} d r d(z, \phi) ; \\
& \int_{\tilde{D}_{Y}} \int_{-\infty}^{\infty} h(r) E_{\chi}(z, \phi ; s) \overline{E_{\chi}(z, \phi ; s)} d r d(z, \phi) \\
& =\int_{\cup M \tilde{D}_{Y}} \int_{-\infty}^{\infty} h(r) E_{\chi}(z, \phi ; s) y^{\dot{s}} e^{i \phi} d r d(z, \phi) \\
& =\int_{0}^{Y} \int_{0}^{1} \int_{0}^{\pi} \int_{-\infty}^{\infty} h(r) E_{x}(z, \phi ; s) y^{\bar{s}-2} e^{i \phi} d r d \phi d x d y
\end{aligned}
$$




$$
\begin{aligned}
& -\int_{\substack{\cup M \tilde{D}_{Y} \\
M \in \Gamma_{\infty} \backslash \Gamma, M \neq I}} \int_{-\infty}^{\infty} h(r) E_{\chi}(z, \phi ; s) y^{\tilde{s}} e^{i \phi} d r d(z, \phi) \\
& =\pi \int_{0}^{Y} \int_{-\infty}^{\infty} h(r)\left(y^{s}+\varphi(s) y^{1-s}\right) y^{\bar{s}-2} d r d y \\
& -\int_{\tilde{D}_{Y^{\prime}}} \int_{-\infty}^{\infty} h(r) E_{\chi}(z, \phi ; s)\left\{\overline{E_{\chi}(z, \phi ; s)}-e^{i \phi} y^{s}\right\} d r d(z, \phi) \\
& =\pi \int_{0}^{Y} \int_{-\infty}^{\infty} h(r) y^{2 \sigma-2} d r d y+\pi \int_{0}^{Y} \int_{-\infty}^{\infty} h(r) \varphi_{x}(s) y^{-1-2 i r} d r d y \\
& -\int_{\tilde{D}_{Y^{\prime}}} \int_{-\infty}^{\infty} h(r)\left\{E_{\mathrm{x}}(z, \phi ; s)-e^{-i \phi}\left(y^{s}+\varphi_{\chi}(s) y^{1-s}\right)\right\} \\
& \left.\times\left\{\overline{E_{\chi}(z, \phi ; s}\right)-e^{i \phi}\left(y^{\bar{s}}+\overline{\varphi_{x}(s)} y^{1-\bar{s}}\right)\right\} d r d(z, \phi) \\
& -\pi \int_{Y}^{\infty} \int_{-\infty}^{\infty} h(r) \overline{\varphi_{x}(s)} y^{-1+2 i r} d r d y-\pi \int_{Y}^{\infty} \int_{-\infty}^{\infty} h(r)\left|\varphi_{x}(s)\right|^{2} d r d y \text {. }
\end{aligned}
$$

Therefore we have

$$
\begin{aligned}
\int_{\tilde{D}_{Y}} \tilde{H}_{\delta}(z, \phi ; z, \phi) d(z, \phi)=\frac{1}{4 \pi} \lim _{\sigma \rightarrow \frac{1}{2}}\left\{\int_{0}^{Y} \int_{-\infty}^{\infty} h(r) y^{2 \sigma-2} d r d y\right. \\
\quad-\int_{Y}^{\infty} \int_{-\infty}^{\infty} h(r)\left|\varphi_{x}(s)\right|^{2} y^{-2 \sigma} d r d y+\int_{0}^{Y} \int_{-\infty}^{\infty} h(r) \varphi_{x}(s) y^{-1-2 i r} d r d y \\
\left.\quad-\int_{Y}^{\infty} \int_{-\infty}^{\infty} h(r) \overline{\varphi_{x}(s)} y^{-1+2 i r} d r d y\right\}+o(1),
\end{aligned}
$$

as $Y \rightarrow \infty$. Here we calculate the integrals appearing in the above expression.

( i ) $\quad \int_{0}^{Y} \int_{-\infty}^{\infty} h(r) y^{2 \sigma-2} d r d y=\frac{Y^{2 \sigma-1}}{2 \sigma-1} \int_{-\infty}^{\infty} h(r) d r$

$$
\begin{gathered}
=\frac{Y^{2 \sigma-1}-Y^{1-2 \sigma}}{2 \sigma-1} \int_{-\infty}^{\infty} h(r) d r+\frac{Y^{1-2 \sigma}}{2 \sigma-1} \int_{-\infty}^{\infty} h(r) d r \\
\int_{-\infty}^{\infty} h(r) d r=2^{2+\delta} \pi \frac{\Gamma\left(\frac{1}{2}\right) \Gamma\left(\frac{1+\delta}{2}\right)}{\Gamma(\delta) \Gamma\left(1+\frac{\delta}{2}\right)} \int_{-\infty}^{\infty}\left|\Gamma\left(\frac{\delta}{2}+i r\right)\right|^{2} d r \\
=2^{2+\delta} \pi \frac{\Gamma\left(\frac{1}{2}\right) \Gamma\left(\frac{1+\delta}{2}\right)}{\Gamma(\delta) \Gamma\left(1+\frac{\delta}{2}\right)} \cdot \sqrt{\pi} \Gamma\left(\frac{\delta}{2}\right) \Gamma\left(\frac{\delta+1}{2}\right) \\
=2^{3} \pi^{5 / 2} \frac{\Gamma\left(\frac{1}{2}\right) \Gamma\left(\frac{1+\delta}{2}\right)}{\Gamma\left(1+\frac{\delta}{2}\right)}
\end{gathered}
$$




$$
\lim _{\sigma \rightarrow \frac{3}{2}} \frac{Y^{2 \sigma-1}-Y^{1-2 \sigma}}{2 \sigma-1}=2 \log Y
$$

(ii) $\quad \int_{0}^{Y} \int_{-\infty}^{\infty} h(r) \varphi_{x}(s) y^{-1-2 i r} d r d y=\int_{0}^{Y} \int_{-\infty}^{\infty} h(r) \varphi_{x}(s) e^{-2 \pi i \cdot \log y} d r d(\log y)$

$$
\begin{aligned}
& =\int_{-\infty}^{\log Y} \int_{-\infty}^{\infty} h(r) \varphi_{x}(s) e^{-i r\left(2 y^{\prime}\right)} d r d y^{\prime} . \\
& =\pi h(0) \varphi_{x}\left(\frac{1}{2}\right)
\end{aligned}
$$

as $Y \rightarrow \infty$. By a similar calculation as in the above,

$$
\int_{Y}^{\infty} \int_{-\infty}^{\infty} h(r) \overline{\varphi_{x}(s)} y^{-1+2 i r} d r d y=\int_{\log Y}^{\infty} \int_{-\infty}^{\infty} h(r) \overline{\varphi_{x}(s)} e^{i r\left(2 y^{\prime}\right)} d r d y^{\prime}
$$

as $Y \rightarrow \infty$. Hence we have

$$
\begin{aligned}
& \lim _{\sigma \rightarrow \frac{1}{2}}\left\{\int_{0}^{Y} \int_{-\infty}^{\infty} h(r) \varphi_{x}(s) y^{-1-2 i r} d r d y-\int_{Y}^{\infty} \int_{-\infty}^{\infty} h(r) \overline{\varphi_{x}(s)} y^{-1+2 i r} d r d y\right\} \\
& \quad=\pi h(0) \varphi_{x}\left(\frac{1}{2}\right)
\end{aligned}
$$

as $Y \rightarrow \infty$. We note that $\varphi_{x}\left(\frac{1}{2}\right)= \pm 1$.

(iii) $\quad-\int_{Y}^{\infty} \int_{-\infty}^{\infty} h(r)\left|\varphi_{X}(s)\right|^{2} y^{-2 \sigma} d r d y+\frac{Y^{1-2 \sigma}}{2 \sigma-1} \int_{-\infty}^{\infty} h(r) d r$

$$
\begin{aligned}
& =-\frac{Y^{1-2 \sigma}}{2 \sigma-1} \int_{-\infty}^{\infty} h(r)\left|\varphi_{x}(s)\right|^{2} d r+\frac{Y^{1-2 \sigma}}{2 \sigma-1} \int_{-\infty}^{\infty} h(r) d r \\
& =-Y^{1-2 \sigma} \int_{-\infty}^{\infty} h(r) \frac{\left|\varphi_{x}(s)\right|^{2}-1}{2 \sigma-1} d r .
\end{aligned}
$$

By the Maass-Selberg relation ([3], [4]), we know that

$$
\left|\varphi_{x}(s)\right| \longrightarrow 1 \text { as } \sigma \longrightarrow \frac{1}{2} ;
$$

and hence

$$
\varphi_{x}\left(\frac{1}{2}+i r\right) \varphi_{x}\left(\frac{1}{2}-i r\right)=1
$$

namely,

$$
\lim _{\sigma \rightarrow \frac{1}{2}} \int_{-\infty}^{\infty} h(r) \frac{\left|\varphi_{x}(s)\right|^{2}-1}{2 \sigma-1} d r=\int_{-\infty}^{\infty} h(r) \frac{\varphi_{x}^{\prime}\left(\frac{1}{2}+i r\right)}{\varphi_{x}\left(\frac{1}{2}+i r\right)} d r
$$


By (i), (ii) and (iii), we obtain for the second half of the expression for $J(\infty)$,

$$
\begin{aligned}
\int_{\tilde{D}_{Y}} \tilde{H}_{\delta}(z, \phi ; z, \phi) d(z, \phi) & \\
= & 2^{2} \pi \frac{\Gamma\left(\frac{1}{2}\right) \Gamma\left(\frac{\delta+1}{2}\right)}{\Gamma\left(1+\frac{\delta}{2}\right)} \log Y-\frac{1}{4 \pi} \int_{-\infty}^{\infty} h(r) \frac{\varphi_{x}^{\prime}\left(\frac{1}{2}+i r\right)}{\varphi_{x}\left(\frac{1}{2}+i r\right)} d r \\
& +\frac{1}{4} h(0) \varphi_{x}\left(\frac{1}{2}\right)+o(1)
\end{aligned}
$$

as $Y \rightarrow \infty$. Summing up the above results (A) and (B), we obtain

$$
\begin{aligned}
J(\infty)= & 2^{2} \pi \frac{\Gamma\left(\frac{1}{2}\right) \Gamma\left(\frac{\delta+1}{2}\right)}{\Gamma\left(1+\frac{\delta}{2}\right)}(C+\log 2)+\frac{1}{4 \pi} \int_{-\infty}^{\infty} h(r) \frac{\varphi_{x}^{\prime}\left(\frac{1}{2}+i r\right)}{\varphi_{x}\left(\frac{1}{2}+i r\right)} d r \\
& -\frac{1}{4} h(0) \varphi_{x}\left(\frac{1}{2}\right) \\
& +2 \pi\left\{-\sum_{k=0}^{\infty}\left(\begin{array}{c}
-\delta / 2-1 \\
k
\end{array}\right) \frac{1}{\left(\frac{1}{2}+k\right)^{2}}\right. \\
& \left.+\sum_{k=0}^{\infty}\left(\begin{array}{c}
-\delta / 2-1 \\
k
\end{array}\right) \frac{1}{\left(\frac{\delta+9}{2}+k\right)^{2}}\right\} .
\end{aligned}
$$

(C) We shall now calculate the limit $\lim _{\delta \rightarrow 0} \delta J(\infty)$.

(iv) we use the formula:

$$
\left(\begin{array}{c}
-\delta / 2-1 \\
k
\end{array}\right)=(-1)^{k} \frac{\Gamma\left(k+\frac{\delta}{2}+1\right)}{k ! \Gamma\left(\frac{\delta}{2}+1\right)}
$$

Then,

$$
\lim _{\delta \rightarrow 0} \delta\left\{-\sum_{k=0}^{\infty}\left(\begin{array}{c}
-\delta / 2-1 \\
k
\end{array}\right) \frac{1}{\left(\frac{1}{2}+k\right)^{2}}+\sum_{k=0}^{\infty}\left(\begin{array}{c}
-\delta / 2-1 \\
k
\end{array}\right) \frac{1}{\left(\frac{\delta+9}{2}+k\right)^{2}}\right\}
$$




$$
\begin{aligned}
= & -\lim _{\delta \rightarrow 0} \frac{\delta}{\Gamma\left(\frac{\delta}{2}+1\right)^{\infty}} \sum_{k=0}^{\infty} \frac{(-1)^{k} \Gamma\left(k+\frac{\delta}{2}+1\right)}{k !\left(\frac{1}{2}+k\right)^{2}} \\
& +\lim _{\delta \rightarrow 0} \frac{\delta}{\Gamma\left(\frac{\delta}{2}+1\right)^{k}} \sum_{k=0}^{\infty} \frac{(-1)^{k} \Gamma\left(k+\frac{\delta}{2}+1\right)}{k !\left(\frac{\delta+9}{2}+k\right)^{2}} \\
= & -\lim _{\delta \rightarrow 0} \delta \sum_{k=0}^{\infty} \frac{(-1)^{k}}{\left(\frac{1}{2}+k\right)^{2}}+\lim _{\delta \rightarrow 0} \delta \sum_{k=0}^{\infty} \frac{(-1)^{k}}{\left(\frac{9}{2}+k\right)^{2}}=0 .
\end{aligned}
$$

(v) By the expression (2.2) of $h(r)$, we have

$$
\lim _{\delta \rightarrow 0} \delta h(0)=16 \pi^{2}
$$

Therefore

$$
\lim _{\delta \rightarrow 0} \delta\left(-\frac{1}{4} h(0) \varphi_{x}\left(\frac{1}{2}\right)\right)=-4 \pi^{2} \varphi_{x}\left(\frac{1}{2}\right) .
$$

By (2.2), we also have

$$
h(r) \sim \frac{c(\delta)|r|^{\delta}}{|r| e^{\pi|r|}}
$$

as $r \rightarrow \infty$, where $c(\delta)$ is independent of $r$ and $\lim _{\delta \rightarrow 0} c(\delta)$ is finite. On the other hand, if we put

$$
f(r)=\varphi_{x}^{\prime}\left(\frac{1}{2}+i r\right) / \varphi_{x}\left(\frac{1}{2}+i r\right)
$$

then

$$
\begin{aligned}
\lim _{\delta \rightarrow+0} \delta \int_{-\infty}^{\infty} h(r) \frac{\varphi_{x}^{\prime}}{\varphi_{x}}\left(\frac{1}{2}+i r\right) d r \\
=\lim _{\delta \rightarrow+0} \delta\left\{\int_{-\infty}^{-N} h(r) \frac{\varphi_{x}^{\prime}}{\varphi_{x}}\left(\frac{1}{2}+i r\right) d r+\int_{-N}^{N} h(r) \frac{\varphi_{x}^{\prime}}{\varphi_{x}}\left(\frac{1}{2}+i r\right) d r\right. \\
\left.\quad+\int_{N}^{\infty} h(r) \frac{\varphi_{x}^{\prime}}{\varphi_{x}}\left(\frac{1}{2}+i r\right) d r\right\} .
\end{aligned}
$$

Since the function $f(r)$ is bounded on $[-N, N]$, we have

$$
\begin{gathered}
\int_{-N}^{N} h(r) f(r) d r=O\left(\int_{-\infty}^{\infty} h(r) d r\right), \text { i.e. } \\
\lim _{\delta \rightarrow+0} \delta \int_{-N}^{N} h(r) f(r) d r=0
\end{gathered}
$$


Moreover, Since the operator $\tilde{K}_{\delta}$ is completely continuons on $L^{2}(\Gamma \backslash \tilde{S})$, there exists some constant $\delta_{1}$ such that

$$
\int_{N}^{\infty}|r|^{\delta_{1}} \frac{f(r)}{|r| e^{\pi|r|}} d r<+\infty
$$

Then, for any $\delta$ such that $0 \leqq \delta<\delta_{1}$, the function $|r|^{\delta}\left(f(r) /|r| e^{\pi|r|}\right)$ is integrable on $[N, \infty)$ and its convergence is uniform for $\delta$. Thus

$$
\lim _{\delta \rightarrow+0} \delta \int_{N}^{\infty}|r|^{\delta} \frac{f(r)}{|r| e^{\pi|r|}} d r=0
$$

Therefore, we have

$$
\lim _{\delta \rightarrow+0} \delta \int_{-\infty}^{\infty} h(r) \frac{\varphi_{x}^{\prime}}{\varphi_{x}}\left(\frac{1}{2}+i r\right) d r=0 .
$$

Remark 1. The function $\left(\varphi_{x}^{\prime} / \varphi_{x}\right)(1 / 2+i r)$ satisfies

$$
\left|\frac{\varphi_{x}^{\prime}}{\varphi_{x}}\left(\frac{1}{2}+i r\right)\right|<c \log (2+|r|)
$$

for some constant $c$. By this estimation we have again

$$
\lim _{\delta \rightarrow 0} \delta \int_{-\infty}^{\infty} h(r) \frac{\varphi_{x}^{\prime}}{\varphi_{x}}\left(\frac{1}{2}+i r\right) d r=0 .
$$

It is now clear that the above result, combined with the formula (1), proves the following.

THEOREM F. Let $\Gamma$ be a fuchsian group of the first kind containing the element $\left(\begin{array}{rr}-1 & 0 \\ 0 & -1\end{array}\right)(=-I)$ and suppose that $\Gamma$ is reduced at infinity. Let $\chi$ be a one-dimensional unitary representation of $\Gamma$ such that $\chi(-I)=$ $-1, \chi\left(\left(\begin{array}{ll}1 & 1 \\ 0 & 1\end{array}\right)\right)=1$ and $\chi^{2} \neq 1$, and denote by $d_{1}$ the dimension for the linear space consisting of all cusp forms of weight one with respect to $\Gamma$ with $\chi$. Then the dimension $d_{1}$ is given by

$$
d_{1}=\frac{1}{2} \sum_{\{M\}} \frac{\chi(M)}{[\Gamma(M): \pm I]} \frac{\bar{\zeta}}{1-\bar{\zeta}^{2}}+\frac{1}{2} \operatorname{Res}_{s=0} \zeta^{*}(s)-\frac{1}{4} \varphi_{x}\left(\frac{1}{2}\right),
$$

where the sum over $\{M\}$ is taken over the distinct elliptic conjugacy classes of $\Gamma /\{ \pm I\}, \Gamma(M)$ denotes the centralizer of $M$ in $\Gamma, \bar{\zeta}$ is one of the eigenvalues of $M$, and $\zeta^{*}(s)$ denotes the Selberg type zeta-function defined in Section 0 . 
We may call the formula (3.2) a kind of Riemann-Roch type theorem for automorphic forms of weight one.

Example. Let $p$ be a prime number such that $p \equiv 3 \bmod 4,(p \neq 3)$, and let $\Phi_{0}(p)$ be the group generated by the group $\Gamma_{0}(p)$ and the element $K=\left(\begin{array}{cc}0 & -\sqrt{p}^{-1} \\ \sqrt{p} & 0\end{array}\right)$. Let $\varepsilon$ be the Legendre symbol on $\Gamma_{0}(p): \varepsilon(L)=(d / p)$ for $L=\left(\begin{array}{ll}a & b \\ c & d\end{array}\right) \in \Gamma_{0}(p)$. Since $\varepsilon\left(K^{2}\right)=\varepsilon(-I)=-1$, we can define the odd characters $\varepsilon^{ \pm}$on the Fricke group $\Phi_{0}(p)$ such that $\varepsilon^{ \pm}(K)= \pm i$. Then we have

$$
S_{1}\left(\Gamma_{0}(p), \varepsilon\right)=S_{1}\left(\Phi_{0}(p), \varepsilon^{+}\right) \oplus S_{1}\left(\Phi_{0}(p), \varepsilon^{-}\right)
$$

We put

$$
\mu_{1}^{ \pm}=\operatorname{dim} S_{1}\left(\Phi_{0}(p), \varepsilon^{ \pm}\right)
$$

Then

$$
d_{1}=\operatorname{dim} S_{1}\left(\Gamma_{0}(p), \varepsilon\right)=\mu_{1}^{+}+\mu_{1}^{-} .
$$

If $\sigma^{*}(p)$ is the parabolic class number of $\Phi_{0}(p) /\{ \pm I\}$, then $\sigma^{*}(p)=1$. As shown in [6], the contribution from elliptic classes to $\mu_{1}^{ \pm}$is given by

$$
\frac{1}{2} \sum_{\{M\}} \frac{1}{[\Gamma(M): \pm I]} \frac{\bar{\zeta}}{1-\bar{\zeta}^{2}} \varepsilon^{ \pm}(M)=\mp \frac{1}{4} h .
$$

We also have $\varphi_{\varepsilon} \pm(1 / 2)=\mp 1$. Let $P_{\alpha}(\alpha=1,2,3, \cdots)$ be a complete system of representatives of the primitive hyperbolic conjugacy classes in $\Gamma_{0}(p) /\{ \pm I\}$ and let $\lambda_{0, \alpha}$ be the eigenvalue $\left(\lambda_{0, \alpha}>1\right)$ of a representative $P_{\alpha}$. We put

$$
\mathrm{Z}^{*}(s)=\sum_{\alpha=1}^{\infty} \sum_{k=1}^{\infty} \frac{\varepsilon\left(P_{\alpha}\right)^{k} \log \lambda_{0, \alpha}}{\lambda_{0, \alpha}^{k}-\lambda_{0, \alpha}^{-k}}\left|\lambda_{0, \alpha}^{k}+\lambda_{0, \alpha}^{-k}\right|^{-s} .
$$

Then, we have the following formula for $d_{1}$ which is our conclusion

$$
d_{1}=\mu_{1}^{+}+\mu_{1}^{-}=\frac{1}{2} \operatorname{Res}_{s=0} Z^{*}(s) .
$$

Remark 2. For a general discontinuous group $\Gamma$ of finite type containing the element $\left(\begin{array}{rr}-1 & 0 \\ 0 & -1\end{array}\right)$, we obtain, in the same way as in the case of a group reduced at $\infty$, the contribution from parabolic classes to $d_{1}$. 


\section{Addendum}

Taking this opportunity, I would like to comment on our previous paper:

T. Hiramatsu and Y. Mimura,

The modular equation and modular forms of weight one,

Nagoya Math. J., 100 (1985), 145-162.

By an exchange of letters, it has been shown that the paper "Hohere Reziprozitatggesetze und Modulformen von Gewicht Eins, Jour. reine angew. Math., 361 (1985), 11-22”, remarkably overlapping with our one, was written after its author had read a preprint of ours.

\section{REFERENCES}

[1] T. Hiramatsu, On some dimension formula for automorphic forms of weight one I, Nagoya Math. J., 85 (1982), 213-221.

[2] H. Jacquet and R. P. Langlands, Automorphic forms on $G L(2)$, Lecture Notes in Math., 114, Berlin, Heidelberg, New York: Springer 1970.

[3] T. Kubota, Elementary theory of Eisenstein series, Tokyo-New York: Kodansha and Halsted 1973.

[4] A. Selberg, Discontinuous groups and harmonic analysis, in Proc. International Math. Congr., Stockholm, 177-189, 1962.

[5] J.-P. Serre, Modular forms of weight one and Galois representations, in Proc. Symposium on Algebraic Number Fields, 193-268, London: Academic Press 1977.

[ 6 ] T. Hiramatsu, A formula for the dimension of spaces of cusp forms of weight one, to appear in Advanced Studies in Pure Math.

Department of Mathematics

Faculty of Science

Kobe University

Nada-ku, Kobe 657

Japan 